\title{
Effect of Neem Coated Urea on Nitrogen Uptake, Nitrogen Use Efficiency and Yield of Rice under Low Land Ecosystem of Godavari Delta of Andhra Pradesh, India
}

\author{
A. Sireesha*, J. Radha Krishna and P. V. Satyanarayana \\ Regional Agricultural Research Station, Maruteru, West Godavari dt, A.P, India \\ *Corresponding author
}

\section{A B S T R A C T}

\begin{tabular}{|l|} 
Ke y w o r d s \\
$\begin{array}{l}\text { Nitrogen use } \\
\text { efficiency, Neem } \\
\text { coated urea and } \\
\text { Rice yields }\end{array}$ \\
\hline Article Info \\
\hline $\begin{array}{l}\text { Accepted: } \\
20 \text { July } 2020 \\
\text { Available Online: } \\
\text { 10 August } 2020\end{array}$ \\
\hline
\end{tabular}

A field experiment was carried out during the Kharif, 2016 at Regional Agricultural Research Station, Maruteru to study the Response of applied neem coated urea (NCU) on nutrient uptake and nitrogen use efficiency of rice (Oryza sativa $L$ ) in low land rice ecosystem of Godavari delta, West Godavari district of Andhra Pradesh. The experiment was laid out in a Randomized block design with three replications and eight treatments namely i.e. $100 \%$ PU (3 equal splits as basal, tillering and PI stage), $75 \%$ NCU (3 equal splits splits as basal, tillering and PI stage), $100 \%$ NCU (3 equal splits as basal, tillering and PI stage), $125 \%$ NCU (3 equal splits splits as basal, tillering and PI stage), $100 \% \mathrm{NCU}$ (as basal), $100 \% \mathrm{NCU}$ ( 2 splits as $50 \%$ basal and 50\% max. tillering stage) $100 \%$ NCU (2 splits $75 \%$ as basal and $25 \%$ at maximum tillering stage) and Control (No P60 K40).

\section{Introduction}

Rice (Oryza sativa L.) remains the most important staple food on the planet since it feeds about half the population on a daily basis. Fertilizer nitrogen $(\mathrm{N})$ has contributed an estimated $40 \%$ to the increase in per capita food production over the past 50 years (Brown, 1999; Smile, 2002). As nitrogen (N) is major most limiting plant nutrient directly influences the growth, development, yield and quality of crops in different cropping systems.
The efficiency of urea is a serious problem both in direct seeded rice in rainfed system as well as recovery of applied fertilizer $\mathrm{N}$ in flooded rice soils. $\mathrm{N}$ is conventionally applied to the soil at various stages in splits before transplanting to panicle initiation stage. Nitrogen use efficiency in rice rarely exceeds 30- 40 per cent. The improvement in the $\mathrm{N}$ efficiency is, therefore, of prime importance, not only for achieving and sustaining high crop grain yield but also to protect the natural resources from degradation. 
Neem has nitrification inhibiting properties (Deva Kumar and Mukherjee, 1985; Santhi et al., 1986) and neemcake-coated urea shows more effectiveness than prilled urea for rice and other crops. With the current thrust on sustainable agriculture and organic farming, the use of natural products like neem has achieved a great practical significance, especially in augmenting the $\mathrm{N}$-use efficiency which abysmally low, around $20-40 \%$ under our predominantly sub-tropical agriculture. Therefore a field study was undertaken to study the effect of nitrogen levels and the modified urea materials on productivity and nitrogen-use efficiency of lowland transplanted rice.

\section{Materials and Methods}

A field experiment was carried out during the Kharif, 2016 at the Regional Agricultural Research Station, Maruteru to study the Response of applied neem coated urea (NCU) on nutrient uptake and nitrogen use efficiency of rice (Oryza sativa L) in low land rice ecosystem of Godavari delta. The soil of experimental site is represented as a clay loam texture. Initial soil characteristics of top-soil $(0-15 \mathrm{~cm}$ layer) was neutral in reaction $(\mathrm{pH}$ 7.19), electrical conductivity $0.57 \mathrm{dSm}^{-1}$, soil organic carbon $1.15 \%$, available N $149 \mathrm{~kg} / \mathrm{ha}$ (Subbiah and Asija 1956), available P2O5 $66.30 \mathrm{~kg} / \mathrm{ha}$ (Olsen et al., 1954) and available $\mathrm{K} 2 \mathrm{O} 364 \mathrm{~kg} / \mathrm{ha}(\mathrm{N}-\mathrm{N}$ NH4OAc-extractable $\mathrm{K})$. The experiment was laid out in a Randomized block design with three replications and eight treatments $100 \%$ PU (3 equal splits as basal, tillering and PI stage), 75 $\%$ NCU (3 equal splits as basal, tillering and PI stage), $100 \%$ NCU (3 equal splits as basal, tillering and PI stage), $125 \%$ NCU (3 equal splits splits as basal, tillering and PI stage), $100 \%$ NCU (as basal), $100 \%$ NCU (2 splits as $50 \%$ basal and $50 \%$ max. tillering stage) $100 \%$ NCU (2 splits $75 \%$ as basal and $25 \%$ at maximum tillering stage) and Control (No
P60 K40). The variety used in this experiment was MTU-1061 (Amara). Recommended dose of fertilizer for rice in Godavari zone of Andhra Pradesh was 90-60-40 kg NPK per Ha.

\section{Results and Discussion}

Effect of neem coated urea on grain and straw yield of rice

There was a significant increase in the grain and straw yields of rice with an increase in level of nitrogen. There was significant highest grain yield of rice $5733 \mathrm{~kg} / \mathrm{ha}$ was recorded with $125 \%$ neem coated urea which was on par with $100 \%$ and $75 \%$ neem coated urea applied in three equal splits as basal, at maximum tillering stage and Panicle initiation stage (5550 and $5133 \mathrm{~kg} / \mathrm{ha}$ as respectively). Application of coated urea materials, e.g. neemcake-coated urea and neem oil emulsioncoated urea, irrespective of the concentrations, had beneficial effects on grain and straw yields of rice. Singh and \& Singh (1991) and Panigrahi and Dixit (1991) also reported superiority of neem-coated urea materials to the prilled urea (Table 1).

\section{Effect of neem coated urea on nutrient content and uptake of rice}

There was not much variation in nutrient content of rice grain and straw, however, the total $\mathrm{N}, \mathrm{P}$ and $\mathrm{K}$ uptake was significantly higher in $125 \%$ NCU (3 splits). In case of nitrogen, maximum uptake was found in 125 $\%$ NCU (3 splits) $101.38 \mathrm{~kg} \mathrm{ha}^{-1}$ followed by $100 \%$ NCU (3 split) $100.53 \mathrm{~kg} \mathrm{ha}^{-1}, 75 \%$ NCU (3 splits) $95.17 \mathrm{~kg} \mathrm{ha}^{-1}$ and lowest from control (No nitrogen) $35.36 \mathrm{~kg} \mathrm{ha}{ }^{-1}$. Phosphorus and Potassium uptake also followed the same trend. With regard to different $\mathrm{N}$ source, neem cake blended urea recorded the highest uptake and prilled urea recorded the lowest uptake. Upadhyay and 
Tripathi (2000), Shivay et al., (2000) and Thind et al., (2010) also found superiority of NCU over ordinary urea in $\mathrm{N}$ uptake and nitrogen use efficiencies (Table 2-4).

\section{Effect of neem coated urea on nitrogen use efficiency}

Nitrogen use efficiency (NUE) followed the similar trend (Table 5) as that of grain yield and recorded higher NUE in $125 \%$ NCU (3split) $33.01 \%$ followed by $100 \% \mathrm{NCU}$ (3split) $32.59 \%, 75 \%$ NCU (3 split) $29.41 \%$ and $100 \%$ prilled urea $20.68 \%$. With regard to NUE, Khanna et al., (2000) found that neem based product coated urea on rice produced the maximum grain yield and $\mathrm{N}$ use efficiency $(30.21 \mathrm{~kg}$ grain $/ \mathrm{kg} \mathrm{N})$, which was significantly superior to prilled urea (PU), and higher N use efficiency. Similarly Singh and
Shivay (2003) reported that coated urea with neem formulations not only increased the grain yield, but also increased NUE and apparent N recovery. Kumar et al., (2011) and Pushpanathan et al., (2005) also reported similar finding.

\section{Effect of neem coated urea on soil nutrient status}

Post harvest soil analysis data revealed that, soil nitrogen status was high with the treatment receiving 125\% Nitrogen applied as neem coated urea followed by $100 \%$ NCU. Control plot recorded the lowest soil nitrogen status. Raj et al., (2014) found that neem cake blended urea maintained high available $\mathrm{N}$ status in the soil compared to other slow release forms of urea. Prilled urea maintained lower $\mathrm{N}$ status.

Table.1 Effect of application of neem coated urea on grain and straw yield $(\mathrm{kg} / \mathrm{ha})$ during kharif-2016

\begin{tabular}{|c|c|c|c|c|}
\hline \multicolumn{2}{|c|}{ Treatments } & \multirow{2}{*}{$\begin{array}{c}\text { Grain yield } \\
\text { Kg/ha } \\
4850\end{array}$} & \multirow{2}{*}{$\begin{array}{c}\text { Straw yield } \\
\text { Kg/ha }\end{array}$} & \multirow{2}{*}{$\begin{array}{c}\text { Panicles/m2 } \\
217\end{array}$} \\
\hline $\mathrm{T} 1$ & $\begin{array}{l}\text { 100\% Prilled Urea (PU) - 3Splits(Basal, max. } \\
\text { tillering and PI ) }\end{array}$ & & & \\
\hline $\mathrm{T} 2$ & $\begin{array}{l}\text { 75\% Neem coated urea (NCU) - } 3 \text { Splits (Basal, } \\
\text { max. tillering and PI) }\end{array}$ & 5133 & 6811 & 226 \\
\hline $\mathrm{T} 3$ & $\begin{array}{l}\text { 100\% NCU (3 Splits - Basal, max. tillering and } \\
\text { PI) }\end{array}$ & 5550 & 7190 & 242 \\
\hline $\mathrm{T} 4$ & $\begin{array}{l}\text { 125\% NCU(3 Splits - Basal, max. tillering and } \\
\text { PI) }\end{array}$ & 5733 & 7393 & 249 \\
\hline T5 & $100 \%$ NCU (Full Basal) & 3767 & 5460 & 182 \\
\hline T6 & $\begin{array}{l}100 \% \text { NCU ( } 2 \text { Splits - 50\%- Basal + 50\%- max. } \\
\text { tillering) }\end{array}$ & 3833 & 5542 & 207 \\
\hline $\mathrm{T} 7$ & $\begin{array}{l}100 \% \text { NCU ( } 2 \text { Splits }-75 \% \text { Basal+25\% max. } \\
\text { tillering) }\end{array}$ & 4367 & 6199 & 215 \\
\hline \multirow[t]{4}{*}{$\mathrm{T} 8$} & $\begin{array}{l}\text { Control (No nitrogen fertilizer application (only } \\
\mathrm{P} \text { and } \mathrm{K} \text { ) }\end{array}$ & 2400 & 3074 & 151 \\
\hline & Mean & 3959 & 5304 & 187 \\
\hline & C.V $(\%)$ & 14.3 & 11.0 & 16.6 \\
\hline & C.D $(0.05)$ & 588 & 719 & 21.6 \\
\hline
\end{tabular}


Table.2 Effect of application of neem coated urea on N, P and K content in grain and straw during kharif-2016

\begin{tabular}{|c|c|c|c|c|c|c|c|}
\hline \multirow{3}{*}{\multicolumn{2}{|c|}{ Treatments }} & \multicolumn{6}{|c|}{ Content (\%) } \\
\hline & & \multicolumn{3}{|c|}{ Grain } & \multicolumn{3}{|c|}{ Straw } \\
\hline & & $\mathbf{N}$ & $\mathbf{P}$ & $\mathbf{K}$ & $\mathbf{N}$ & $\mathbf{P}$ & $\mathbf{K}$ \\
\hline $\mathrm{T} 1$ & $\begin{array}{l}100 \% \text { Prilled Urea (PU) - 3Splits(Basal, max. } \\
\text { tillering and PI ) }\end{array}$ & 0.95 & 0.28 & 0.24 & 0.40 & 0.14 & 1.23 \\
\hline $\mathrm{T} 2$ & $\begin{array}{l}75 \% \text { Neem coated urea (NCU) - } 3 \text { Splits (Basal, } \\
\text { max. tillering and PI) }\end{array}$ & 0.96 & 0.28 & 0.23 & 0.43 & 0.14 & 1.20 \\
\hline T3 & 100\% NCU (3 Splits - Basal, max. tillering and PI) & 1.09 & 0.30 & 0.24 & 0.45 & 0.16 & 1.21 \\
\hline $\mathrm{T} 4$ & 125\% NCU(3 Splits - Basal, max. tillering and PI) & 1.03 & 0.29 & 0.24 & 0.48 & 0.16 & 1.22 \\
\hline T5 & $100 \%$ NCU (Full Basal) & 0.87 & 0.28 & 0.23 & 0.41 & 0.14 & 1.17 \\
\hline T6 & $\begin{array}{l}100 \% \text { NCU ( } 2 \text { Splits }-50 \% \text { - Basal }+50 \%-\text { max. } \\
\text { tillering) }\end{array}$ & 0.83 & 0.28 & 0.24 & 0.32 & 0.14 & 1.20 \\
\hline $\mathrm{T} 7$ & $\begin{array}{l}100 \% \text { NCU ( } 2 \text { Splits }-75 \% \text { Basal+25\% max. } \\
\text { tillering) }\end{array}$ & 0.99 & 0.28 & 0.21 & 0.41 & 0.16 & 1.18 \\
\hline \multirow[t]{4}{*}{$\mathrm{T} 8$} & $\begin{array}{l}\text { Control (No nitrogen fertilizer application (only } \mathrm{P} \\
\text { and } \mathrm{K} \text { ) }\end{array}$ & 0.60 & 0.30 & 0.24 & 0.35 & 0.14 & 1.19 \\
\hline & Mean & 0.81 & 0.25 & 0.21 & 0.36 & 0.13 & 1.07 \\
\hline & C.V $(\%)$ & 6.74 & 14.34 & 16.10 & 13.85 & 18.27 & 6.34 \\
\hline & C.D $(0.05)$ & 0.10 & 0.06 & 0.06 & 0.09 & 0.04 & 0.12 \\
\hline
\end{tabular}

Table.3 Effect of application of neem coated urea on N, P and K uptake by grain and straw during kharif-2016

\begin{tabular}{|c|c|c|c|c|c|c|c|}
\hline \multirow{2}{*}{\multicolumn{2}{|c|}{ Treatments }} & \multicolumn{6}{|c|}{ Nutrient Uptake (kg/ha) } \\
\hline & & \multicolumn{3}{|c|}{ Grain } & \multicolumn{3}{|c|}{ Straw } \\
\hline & & \multirow{2}{*}{$\begin{array}{c}\mathbf{N} \\
50.88\end{array}$} & \multirow{2}{*}{$\begin{array}{c}\mathbf{P} \\
15.00\end{array}$} & \multirow{2}{*}{$\frac{\mathbf{K}}{12.66}$} & \multirow{2}{*}{$\frac{\mathbf{N}}{25.83}$} & \multirow{2}{*}{$\begin{array}{l}\mathbf{P} \\
9.03\end{array}$} & \multirow{2}{*}{$\frac{\mathbf{K}}{78.66}$} \\
\hline $\mathrm{T} 1$ & $\begin{array}{l}100 \% \text { Prilled Urea (PU) - 3Splits(Basal, } \\
\text { max. tillering and PI ) }\end{array}$ & & & & & & \\
\hline $\mathrm{T} 2$ & $\begin{array}{l}75 \% \text { Neem coated urea }(\mathrm{NCU})-3 \text { Splits } \\
\text { (Basal, max. tillering and PI) }\end{array}$ & 55.79 & 16.18 & 13.47 & 29.38 & 9.35 & 81.40 \\
\hline $\mathrm{T} 3$ & $\begin{array}{l}100 \% \text { NCU (3 Splits - Basal, max. tillering } \\
\text { and PI) }\end{array}$ & 67.98 & 18.56 & 15.24 & 32.55 & 11.82 & 87.46 \\
\hline $\mathrm{T} 4$ & $\begin{array}{l}125 \% \text { NCU(3 Splits - Basal, max. tillering } \\
\text { and PI) }\end{array}$ & 66.19 & 18.60 & 14.97 & 35.19 & 11.89 & 90.53 \\
\hline T5 & $100 \%$ NCU (Full Basal) & 38.57 & 12.52 & 10.23 & 22.40 & 7.66 & 64.24 \\
\hline T6 & $\begin{array}{l}100 \% \text { NCU ( } 2 \text { Splits - 50\%- Basal }+50 \%- \\
\text { max. tillering) }\end{array}$ & 37.59 & 12.78 & 10.67 & 17.77 & 7.76 & 66.39 \\
\hline $\mathrm{T} 7$ & $\begin{array}{l}100 \% \text { NCU ( } 2 \text { Splits }-75 \% \text { Basal+25\% } \\
\text { max. tillering) }\end{array}$ & 49.73 & 14.24 & 10.69 & 25.25 & 9.89 & 73.11 \\
\hline \multirow[t]{4}{*}{$\mathrm{T} 8$} & $\begin{array}{l}\text { Control (No nitrogen fertilizer application } \\
\text { (only P and K) }\end{array}$ & 20.80 & 10.29 & 8.13 & 14.56 & 5.67 & 49.31 \\
\hline & Mean & 43.06 & 13.13 & 10.67 & 22.55 & 8.12 & 65.68 \\
\hline & C.V $(\%)$ & 16.32 & 19.17 & 20.94 & 16.27 & 21.97 & 12.29 \\
\hline & C.D (0.05) & 12.27 & 4.40 & 3.91 & 6.41 & 3.12 & 14.10 \\
\hline
\end{tabular}


Table.4 Effect of application of neem coated urea on soil nutrient status during kharif-2016

\begin{tabular}{|c|c|c|c|c|c|c|c|}
\hline \multirow{2}{*}{\multicolumn{2}{|c|}{ Treatments }} & \multirow{3}{*}{$\begin{array}{l}\mathbf{p H} \\
6.24\end{array}$} & \multirow{3}{*}{$\begin{array}{r}\begin{array}{c}\text { E.C } \\
(\mathbf{d S} / \mathbf{m})\end{array} \\
0.50\end{array}$} & \multirow{3}{*}{$\begin{array}{l}\mathrm{OC} \\
(\%) \\
1.23\end{array}$} & \multirow{2}{*}{\multicolumn{3}{|c|}{ Available nutrients (kg/ha) }} \\
\hline & & & & & & & \\
\hline $\mathrm{T} 1$ & $\begin{array}{l}100 \% \text { Prilled Urea (PU) - 3Splits(Basal, } \\
\text { max. tillering and PI ) }\end{array}$ & & & & $\mathbf{N}$ & $\begin{array}{r}\mathbf{P}_{\mathbf{2}} \mathbf{O}_{5} \\
57.4\end{array}$ & $\begin{array}{c}\mathbf{K}_{2} \mathbf{O} \\
305\end{array}$ \\
\hline $\mathrm{T} 2$ & $\begin{array}{l}75 \% \text { Neem coated urea (NCU) - } 3 \text { Splits } \\
\text { (Basal, max. tillering and PI) }\end{array}$ & 6.19 & 0.49 & 1.26 & 204 & 57.5 & 326 \\
\hline $\mathrm{T} 3$ & $\begin{array}{l}100 \% \text { NCU ( } 3 \text { Splits - Basal, max. } \\
\text { tillering and PI) }\end{array}$ & 6.46 & 0.46 & 1.18 & 213 & 57.3 & 325 \\
\hline $\mathrm{T} 4$ & $\begin{array}{l}125 \% \text { NCU(3 Splits - Basal, max. } \\
\text { tillering and PI) }\end{array}$ & 6.35 & 0.51 & 1.27 & 217 & 58.2 & 317 \\
\hline T5 & $100 \%$ NCU (Full Basal) & 6.46 & 0.54 & 1.21 & 200 & 57.5 & 315 \\
\hline T6 & $\begin{array}{l}100 \% \text { NCU ( } 2 \text { Splits }-50 \% \text { - Basal }+ \\
50 \% \text { - max. tillering) }\end{array}$ & 6.37 & 0.48 & 1.20 & 208 & 57.6 & 304 \\
\hline $\mathrm{T} 7$ & $\begin{array}{l}100 \% \text { NCU ( } 2 \text { Splits }-75 \% \text { Basal }+25 \% \\
\text { max. tillering) }\end{array}$ & 6.27 & 0.50 & 1.23 & 204 & 54.7 & 308 \\
\hline \multirow[t]{4}{*}{$\mathrm{T} 8$} & $\begin{array}{l}\text { Control (No nitrogen fertilizer application } \\
\text { (only P and K) }\end{array}$ & 6.16 & 0.48 & 1.15 & 175 & 52.4 & 304 \\
\hline & Mean & 6.31 & 0.50 & 1.08 & 180 & 50.3 & 278 \\
\hline & C.V $(\%)$ & 2.39 & 13.28 & 5.75 & 7.43 & 6.10 & 8.0 \\
\hline & C.D $(0.05)$ & 0.26 & 0.11 & 0.11 & 23.5 & 5.36 & 38.8 \\
\hline
\end{tabular}

Table.5 Effect of application of neem coated urea on total nitrogen uptake $(\mathrm{kg} / \mathrm{ha})$ and nitrogen use efficiency (\%) in rice during kharif-2016

\begin{tabular}{|c|c|c|c|}
\hline S.No & Treatments & $\begin{array}{l}\text { Total Nitrogen } \\
\text { Uptake (kg/ha) }\end{array}$ & $\begin{array}{c}\text { Nitrogen Use Efficiency } \\
(\%)\end{array}$ \\
\hline T1 & $\begin{array}{l}\text { 100\% Prilled Urea (PU) - } \\
\text { 3Splits(Basal, max. tillering and PI ) }\end{array}$ & 76.71 & 20.68 \\
\hline T2 & $\begin{array}{l}75 \% \text { Neem coated urea (NCU) }-3 \\
\text { Splits (Basal, max. tillering and PI) }\end{array}$ & 95.17 & 29.41 \\
\hline T3 & $\begin{array}{l}100 \% \text { NCU ( } 3 \text { Splits - Basal, max. } \\
\text { tillering and PI) }\end{array}$ & 100.53 & 32.59 \\
\hline T4 & $\begin{array}{l}125 \% \text { NCU( } 3 \text { Splits - Basal, max. } \\
\text { tillering and PI) }\end{array}$ & 101.38 & 33.01 \\
\hline T5 & $100 \%$ NCU (Full Basal) & 60.97 & 12.81 \\
\hline T6 & $\begin{array}{l}100 \% \text { NCU (2 Splits - 50\%- Basal + } \\
50 \% \text { - max. tillering) }\end{array}$ & 55.36 & 10.00 \\
\hline T7 & $\begin{array}{l}100 \% \text { NCU }(2 \text { Splits } \\
\text { Basal }+25 \% \text { max. tillering })\end{array}$ & 74.98 & 19.81 \\
\hline T8 & $\begin{array}{l}\text { Control (No nitrogen fertilizer } \\
\text { application (only } \mathrm{P} \text { and } \mathrm{K} \text { ) }\end{array}$ & 35.36 & -- \\
\hline & Mean & 73.81 & 19.22 \\
\hline & C.V $(\%)$ & 10.71 & 9.54 \\
\hline & C.D (0.05) & 11.26 & 3.11 \\
\hline
\end{tabular}


In conclusion, the application of neem coated urea significantly increased the grain yield of rice as compared to control (no nitrogen) and prilled urea. Total uptake of Nitrogen, Phosphorus and Potassium by plant was maximum in $125 \%$ NCU (3split), followed by $100 \%$ NCU (3 splits) and minimum was noticed under control. Nitrogen use efficiency was found to be high under the treatments received neem coated urea.

\section{References}

Brown, L. R. 1999. A world watches Institute Report on Progress toward a sustainable society, eds. L. R. Brown, C. Flavin, and $\mathrm{H}$. Hench, 115-132.

Devakumar, C. and Mukcrjce, S.K. 1985. Nitrification retardation by neemproducts. Neem newsletter. 2: 11 - 14.

Khanna P, Pandey N, Tripathi RS. Relative performance of soil FYM conditioned and neem based product coated urea on grain yield and NUE of transplanted rice in Vertisols. Agricultural Sc. Digest. 2000; 20(4):259-260.

Kumar Dinesh, Kumar Deva C, Kumar Rajesh, Das Aurovinda, Panneer Selvam P, Shivay YS. Relative efficiency of prilled urea coated with major neem (Azadirachta indica A. Juss) oil components in lowland irrigated rice of the Indo-Gangetic plains. Archives of Agronomy and Soil Science. 2011; 57:61-74.

Olsen SR, Cole CV, Watanabe FS, Dean LA. Estimation of available phosphorus in soil by extraction with sodium bicarbonate. USDA, Circ. 1954, 939.

Panigrahi R.K. and Dixit. L. 199 1. Relative efficiency of slow-release nitrogenous fertilizers in rainfed rice (Oryza sativa). Indian journal of Agronomy 36: 401 403.

Prasad, R.. Devakumar. C. and Shivay. Y.S. 1993.Neem Research and Development.
Randhawa, N.S. and Parmar B.S. Santhi. S.R.. Palaniappan, Sp. and (Eds). Society of Pesticide Science, India.

Pushpanathan KR, Vijayakumar M, Siddeswaran K. Effect of forms of fertilizer nitrogen and timing of application on growth and yield of rice (Oryza sativa l.) Agriculture Review. 2005; 2:153-156.

Santhi. S.R.. Palaniappan, Sp. and Purushottaman, D. 1986. Influence of neern leaf on nitrification in a lowland rice inhibitors for agriculture, health and the environ- soil. Plalzt and Soil 93 : 133-135

Shivay YS, Prasad R, Singh S. Effect of nitrogen levels and neem-oil emulsions coated urea on growth, yield attributes and yield of wetland rice. In: Extended Summary of International Conference on Managing Natural Resources New Delhi ICAR. 2000; 3:1340-1342.

Singh. G. and Singh, O.P. 199 1. Effect of coated urea materials on rainfed low land transplanted rice (Oryza sativa) and their residual effect on wheat. Indian journal of Agronomy (suppl.): 22 $1-223$.

Smil, V. 2002. Nitrogen and food production proteins for human diets. Ambio 31: 126-131

Subbiah BV, Asija GL. A rapid procedure for determination of available nitrogen status in soil. Current Science. 1956; 25:259-260.

Thind HS, Singh Bijay Pannu, Singh RPS, Yadvinder Singh, Varinderpal Gupta, Vashistha RK. Relative performance of neem (Azadirachta indica) coated urea vis-à-vis ordinary urea applied to rice on the basis of soil test or following need based nitrogen management using leaf colour chart. Nutrient Cycling in Agro ecosystems. 2010; 87:1-8.

Upadhyay SK, Tripathi KS. Response of 
prilled and neem extract coated urea application timings to rice (Oryza sativa), Agric. Sci. Digest. 2000; 20(2):84-86.

\section{How to cite this article:}

Sireesha, A., J. Radha Krishna and Satyanarayana, P.V. 2020. Effect of Neem Coated Urea on Nitrogen Uptake, Nitrogen Use Efficiency and Yield of Rice under Low Land Ecosystem of Godavari Delta of Andhra Pradesh. Int.J.Curr.Microbiol.App.Sci. 9(08): 2086-2091. doi: https://doi.org/10.20546/ijcmas.2020.908.237 